20

\title{
Использование спектральной эллипсометрии и спектроскопии комбинационного рассеяния света в скрининговой диагностике колоректального рака
}

\author{
(C) В.Н. Кручинин ${ }^{1}$, М.В. Кручинина ${ }^{2,3}$, Я.И. Прудникова ${ }^{2}$, Е.В. Спесивцев ${ }^{1}$, С.В. Рыхлицкий ${ }^{1}$, \\ В.А. Володин ${ }^{1,4}$, С.В. Шеховцов ${ }^{5}$, С.Е. Пельтек ${ }^{5}$ \\ ${ }^{1}$ Институт ффизики полупроводников им. А.В. Ржанова Сибирского отделения РАН, \\ 630090 Новосибирск, Россия \\ ${ }^{2}$ НИИ терапии и профилактической медицины - филиал ФГБНУ „Федеральный исследовательский центр Институт \\ цитологии и генетики Сибирского отделения РАН“", \\ 630089 Новосибирск, Россия \\ ${ }^{3}$ Новосибирский государственный медицинский университет, \\ 630091 Новосибирск, Россия \\ ${ }^{4}$ Новосибирский государственный университет, \\ 630090 Новосибирск, Россия \\ ${ }^{5}$ Институт цитологии и генетики Сибирского отделения РАН, \\ 630090 Новосибирск, Россия \\ e-mail: vladd.kruch@yandex.ru
}

Поступила в редакцию 04.10.2018 г.

В окончательной редакции 06.11.2018 г.

Принята к публикации 12.02.2019 г.

С помощью спектроскопии комбинационного рассеяния (КР) и измерений состояния поляризованного света вблизи условий наблюдения поверхностного плазмонного резонанса (ППР) проведен диагностический пилотный мониторинг групп здоровых пациентов и больных колоректальным раком (КРР) при различных стадиях развития заболевания. Для диагностики использовалась реакция антигенов сыворотки крови с антителами к сывороточной М2-пируваткиназе (М2-ПК) вблизи условий наблюдения ППР, для чего в работе использовался созданный в ИФП СО РАН спектральный эллипсометрический комплекс „ЭллипсСПЭК“, обладающий высокими чувствительностью, точностью и неразрушающим характером воздействия на исследуемый образец. Интенсивности пиков при 1005, 1157 и $1520 \mathrm{~cm}^{-1}$ в спектрах КР у больных КРР по сравнению со здоровыми лицами так же, как и интенсивность равновесного плазмонного сигнала оказались достоверно ниже, коррелируя со стадией заболевания и наличием метастазов, что позволяет рассматривать данные оптические методы исследования сыворотки крови как перспективные диагностические подходы при диагностике КРР в том числе на ранних стадиях развития болезни.

Ключевые слова: эллипсометрия, поверхностный плазмонный резонанс, спектроскопия комбинационного рассеяния света, сыворотка крови, диагностика, колоректальный рак.

DOI: $10.21883 /$ OS.2019.07.47946.295-18

\section{Введение}

Оптические методы исследования занимают в биомедицинских исследованиях одно из ключевых мест благодаря их чувствительности, возможности работы с жидкими средами и неразрушающему характеру воздействия на исследуемый объект [1]. Бурное развитие биологии и медицины в последние десятилетия определило необходимость создания аналитической и диагностической аппаратуры нового поколения, обеспечивающей возможность изучения реакций биоорганических объектов, находящихся в жидких средах организма в сверхмалых концентрациях $\left(<10^{-12} \mathrm{M}\right)$. Традиционные оптические методы, используемые для исследования биоорганических объектов, такие как спектроскопия комбинационного рассеяния света, ИК спектроскопия, эллипсометрия, спектрофотометрия, спектрофлуоромет- рия и др., требуют модернизации и внедрения новых методик измерения для повышения чувствительности аппаратуры $[2,3]$.

Одним из физических приемов повышения чувствительности оптического детектирования является измерение вблизи условий наблюдения поверхностного плазмонного резонанса (ППР) при условии полного внутреннего отражения [4]. Для реализации таких измерений используют специальные устройства, такие, например, как ячейка Кречмана [4-6]. Это обеспечивает беспрецедентно высокую чувствительность к особенностям состояния пограничной фазы на поверхности резонансной среды (золото) и в приповерхностной области, в качестве которой могут выступать растворы биоорганических молекул (сыворотка крови). Чувствительность к измеряемым параметрам при этом увеличивается на 2-3 порядка. Так, при эллипсометрических измерениях 
удается зафиксировать сдвиг показателя преломления раствора сыворотки крови до $\Delta n \sim 10^{-6}$. Данный прием с успехом использовался в последние два-три десятка лет $[6,7]$, на его основе созданы промышленные образцы биоорганических анализаторов [8].

Рак толстой кишки является одним из самых часто выявляемых онкологических заболеваний и по распространенности в мире среди онкологических заболеваний занимает третье место у мужчин и второе - у женщин $[9,10]$. Надежная диагностика позволяет обнаружить опухоль на ранних стадиях, оценить тяжесть течения болезни и начать своевременное лечение. Для осуществления ранней онкологической диагностики в настоящее время используют высокоспецифические реакции типа „антиген-антитело“, в частности, реакцию антигенов сыворотки крови пациента с моноклональными антителами к опухолевой М2-пируваткиназе (М2-ПК) [11]. При использовании диагностики ППР антитела предварительно иммобилизируют на поверхности аналитических пластин, покрытых резонансным слоем металла (золото).

Цель данного исследования - на базе спектрального эллипсометрического комплекса с возможностью проведения измерений вблизи наблюдения ППР и спектроскопии комбинационного рассеяния провести пилотный диагностический мониторинг образцов растворов сыворотки крови групп здоровых людей и больных колоректальным раком (КРР) на разных стадиях развития заболевания с последующим сравнительным анализом.

\section{Методы исследований}

\section{Группы пациентов и образцы сыворотки крови}

Обследованы 68 человек: 19 условно здоровых лиц (средний возраст $53 \pm 8$ лет) без онкологической патологии и 49 пациентов (средний возраст $52 \pm 9$ лет, 22 женщины, 27 мужчин) с КРР различных локализаций. Гистологически у всех пациентов диагносцирована аденокарцинома различной степени дифференцирования с преобладанием умеренной.

Пациенты были разделены на 3 группы в зависимости от локализации метастазов: в 1-ю группу вошли 15 пациентов с местно-регионарным КРР (7 из них имели вторую, 8 - третью стадию заболевания), 2-ю группу составили 18 больных с метастазами только в печень (12 из них имели одиночные метастазы, остальные множественные, размеры узлов варьировали от 16 до $92 \mathrm{~mm}$ в диаметре), 3-я группа включала 16 пациентов с внепеченочными метастазами (в том числе в надключичные лимфоузлы, легкие, кости, головной мозг). Таким образом, пациенты 2-й и 3-й групп имели четвертую стадию КРР. Три пациента $(20 \%)$ из первой, шесть $(33 \%)$ из второй и одиннадцать $(69 \%)$ из третьей групп подвергались воздействию адъювантной полихимиотерапии в течение трех месяцев.

Использовалась сыворотка крови обследуемых, полученная центрифугированием цельной крови, взятой натощак, со скоростью $2000 \mathrm{rpm}$ в течение $20 \mathrm{~min}$. Для дальнейших исследований сыворотка крови при необходимости разбавлялась фосфатным буфером. В работе использовался раствор сыворотки крови 1:250.

\section{Эллипсометрия. Анализируемая система}

Высокоспецифичные моноклональные антитела AntiPKM2 antibody [EPR10138(B)], (Abcam RabMab, USA) были иммобилизованы к поверхности золота $(\sim 30-40 \mathrm{~nm})$ по методике, описанной в работе [12]. Тонкий слой золота использовался как резонансная среда и входил в состав а) стеклянных пластин для последующих эллипсометрических измерений („Эллипс СПЭК“, ИФП СО РАН), б) специальных чипов для измерений при помощи оптической биосенсорной системы „ProteOn XPR36“ (BioRad, USA). Измерения в комплексе .Эллипс СПЭК. выполнялись по схеме Кречмана (рис. 1) [13] с использованием стеклянной призмы 2, имеющей оптический контакт с пластиной 3. Пластины с резонансным слоем золота укреплялись внутренней стороной в специальной проточной ячейке 4 , сквозь которую непрерывно циркулировал раствор фосфатного буфера и осуществлялся далее ввод пробы (сыворотка).

Эллипсометр, входящий в состав комплекса, построен на основе оригинальной статической измерительной схемы, разработанной и запатентованной в ИФП СО РАН [14]. В отличие от классической фотометрической схемы данная схема оказывается нечувствительна к колебаниям интенсивности источника света. Детальный анализ рассматриваемой схемы был проведен нами в работе [15], где показано, что для определения эллипсометрических параметров $\Psi$ и $\Delta$ достаточно измерения интенсивностей света в обоих каналах при некоторых фиксированных положениях оптических элементов (конфигурациях). То есть такая схема не требует вращения оптических элементов и модуляции сигнала. Именно

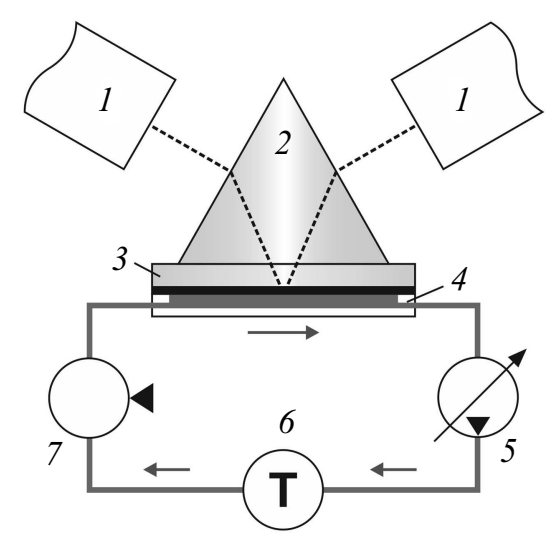

Рис. 1. Схема установки для эллипсометрических измерений вблизи наблюдения ППР: 1 - эллипсометр, 2 - призма, 3 стеклянная пластина с резонансным слоем золота, 4 - кювета с жидкостью, 5 - перистальтический насос, $6-$ термостат, 7 - система ввода пробы. 
благодаря этой особенности удается повысить быстродействие измерительного тракта и отношение сигнала к уровню шумов. Предельное быстродействие, достигнутое при высокой дифференциальной чувствительности, составляет десятки микросекунд. Чувствительность к изменению эллипсометрических параметров составляет $0.0003^{\circ}$. При этом схема позволяет работать при слабой интенсивности зондирующего пучка.

Две величины, измеряемые в эллипсометрии ( $\Psi$ и $\Delta$ - амплитуда и разность фаз $p$ - и $s$-компонент отраженного и падающего лучей поляризованного света), связаны с параметрами отражающей системы уравнением

$$
\operatorname{tg} \Psi e^{i \Delta}=\frac{R_{\mathrm{p}}}{R_{\mathrm{S}}}
$$

где $R_{\mathrm{p}}, R_{\mathrm{s}}$ - комплексные коэффициенты отражения света для волн, поляризованных в плоскости падения и перпендикулярно к ней, зависят от оптических свойств отражающей системы [16] и угла падения луча света.

Условие наблюдения поверхностного плазмонного резонанса можно записать как

$$
\sqrt{\varepsilon_{\text {призма }}} \sin \varphi_{0}=\sqrt{\frac{\varepsilon_{\text {металл }} \varepsilon_{\text {раствор }}}{\varepsilon_{\text {металл }}+\varepsilon_{\text {раствор }}}},
$$

где $\varepsilon_{\text {призма }}, \varepsilon_{\text {металл }}, \varepsilon_{\text {раствор }}$ - диэлектрические константы материала призмы и пластины, слоя металла (золото), раствора (сыворотка в фосфатном буфере) [17]. Изменение диэлектрической константы раствора $\left(\varepsilon_{\text {раствор }}\right)$ в условиях наблюдения ППР (2) приведет к сдвигу пика поглощения ППР а следовательно, к изменению эллипсометрических углов $\Psi$ и $\Delta$ согласно уравнению (1). Экспериментально подтверждено, что измерение в условиях резонансного поглощения света увеличивает чувствительность эллипсометрии на 2-3 порядка и позволяет уверенно определять концентрации белковых молекул в концентрации до десятых долей $\mathrm{ng} / \mathrm{ml}$.

Параллельно детектирование антигенов к высокоспецифичным моноклональным антителам М2-ПК в сыворотках крови, взятых у здоровых людей и больных с КРР, проводили при помощи оптической биосенсорной системы „ProteOn XPR36“ (BioRad, USA), принцип действия которой также основан на измерении амплитуды отраженного поляризованного света в условиях поверхностного плазмонного резонанса. Антитела к опухолевой М2-ПК ковалентно связывали с покрытой золотом поверхностью сенсорного чипа GLC. И в том, и в другом случаях реакция изучалась в сходных физикохимических условиях при условиях наблюдения ППР.

\section{Спектроскопия комбинационного рассеяния}

Спектры комбинационного рассеяния жидких сывороток крови пациентов записывались в диапазоне $500-2000 \mathrm{~cm}^{-1}$ с помощью спектрометра с тройным монохроматором Т64000 (Horiba Jobin Yvon, France) при комнатной температуре. Для возбуждения использовалась линия $\mathrm{Ar}^{+}$-лазера с длиной волны $514.5 \mathrm{~nm}$, спектральное разрешение составляло $1.5 \mathrm{~cm}^{-1}$. В качестве детектора использовалась кремниевая матрица фотоприемников, охлаждаемая жидким азотом. Применялась приставка для микроскопических исследований комбинационного рассеяния света. Мощность лазерного пучка, доходящего до образца, составляла 2-3 $\mathrm{mW}$ (размер пятна 5-6 $\mu \mathrm{m})$. При анализе спектров комбинационного рассеяния оценивали интенсивность пиков в условных единицах.

\section{Статистический анализ}

Статистическая обработка данных выполнена с использованием программы SPSS, ver. 17. Достоверность различия показателей оценивали по критериям Стьюдента-Пирсона в случае, когда распределение подчинялось нормальному закону. В случаях отклонения распределения от нормального закона использовались непараметрические критерии (U-критерий МаннаУитни, Колмогорова-Смирнова). Во всех процедурах статистического анализа критический уровень значимости нулевой гипотезы $(p)$ принимался равным 0.05. Для оценки значимости интенсивности пиков раман-спектров с точки зрения дифференцирования пациентов с КРР с метастазами различных локализаций был использован ROC-анализ.

\section{Результаты и обсуждение}

На рис. 2 представлена кинетика взаимодействия высокоспецифичных моноклональных антител к опухолевой М2-ПК с антигенами сыворотки крови пациентов в исследуемых группах при определении методом спектральной эллипсометрии и средствами оптической биосенсорной системы ProteOn XPR36 вблизи наблюдения ППР. Средний равновесный уровень отклика сывороточной опухолевой М2-ПК, определенный в группе здоровых обследуемых, составил $11.6 \pm 4.5 \mathrm{RU}$ и не зависел от пола $(p=0.51)$ и возраста $(p=0.61)$. Величина $1 \mathrm{RU}$ (resonance unit - единица резонанса) эквивалентна сдвигу, вызываемому связыванием $10^{-12} \mathrm{~g}$ протеина на $1 \mathrm{~mm}^{2}$ чувствительной поверхности, и соответствует $\sim 1.12^{\prime}$ эллипсометрического угла $\Delta$.

Средний равновесный отклик опухолевой М2-ПК оказался равным $67.4 \pm 9.8$ среди пациентов с местнорегионарным КРР (1-я группа), $162.5 \pm 17.8$ - среди больных с КРР с метастазами в печень (2-я группа) и $269.2 \pm 14.6$ - в группе больных с внепеченочными метастазами (3-я группа) ( $p<0.0001-0.05)$, отличаясь в 6-23 раз от такового в группе здоровых (таблица).

Как видно из данных рис. 2, результаты, полученные с помощью спектрального эллипсометрического ППР-комплекса „Эллипс-СПЭК“, оказались сопоставимы с результатами, полученными на приборе „ProteOn 
Средние равновесные уровни сигнала, определенные с помощью эффекта поверхностного плазмонного резонанса, и площади отдельных пиков в спектрах комбинационного рассеяния света у больных с КРР с различной локализацией метастазов и в группе сравнения $(\mathrm{M}+\mathrm{m})$

\begin{tabular}{c|c|c|c|c}
\hline Группы обследованных & Средний уровень сигнала, RU & \multicolumn{3}{|c}{ Площадь пиков КР, arb. units } \\
\cline { 3 - 5 } & & $1005 \mathrm{~cm}^{-1}$ & $1157 \mathrm{~cm}^{-1}$ & $1520 \mathrm{~cm}^{-1}$ \\
\hline Группа сравнения $(n=19)$ & $11.6 \pm 4.5$ & $990 \pm 84$ & $2570 \pm 301$ & $3258 \pm 190$ \\
\hline $\begin{array}{c}\text { 1-я групп, } \\
\text { местно-регионарный КРP }(n=15)\end{array}$ & $67.4 \pm 9.8^{* * * *}$ & $557 \pm 103^{*}$ & $2004 \pm 264^{*}$ & $2404 \pm 162^{* *}$ \\
\hline $\begin{array}{c}\text { 2-я групп, КРР IV ст. } \\
\text { с метастазами в печень }(n=18)\end{array}$ & $162.5 \pm 17.8^{* * * * \wedge \wedge \wedge \wedge}$ & $387 \pm 90^{* * \wedge}$ & $908 \pm 115^{* * \wedge \wedge}$ & $714 \pm 135^{* * * \wedge \wedge \wedge}$ \\
\hline $\begin{array}{c}\text { 3-я группа, КРР IV ст. } \\
\text { с внепеченочными метастазами }(n=16)\end{array}$ & $269.2 \pm 14.6^{* * * * \wedge \wedge \wedge \wedge \$ \$}$ & $129 \pm 78^{* * * \wedge \wedge \wedge \$ \$}$ & $712 \pm 132^{* * * \wedge \wedge \wedge \$ \$}$ & $474 \pm 108^{* * * \wedge \wedge \wedge \$ \$}$
\end{tabular}

Примечание. $M-$ среднее значение, $m-$ стандартная ошибка среднего, статистическая значимость $(p)$ отличия от группы сравнения $\left({ }^{*} p<0.05,{ }^{* *} p<0.02,{ }^{* * *} p<0.0001,{ }^{* * * *} p<0.000001\right)$, статистическая значимость $(p)$ отличия от 1-й групाы $(\wedge p<0.05, \wedge \wedge p<0.02$, $\wedge \wedge \wedge p<0.0001, \wedge \wedge \wedge \wedge p<0.00001)$, статистическая значимость $(p)$ отличия от 2-й группы $(\$-p<0.05, \$ \$ p<0.01, \$ \$ \$ p<0.00001)$.

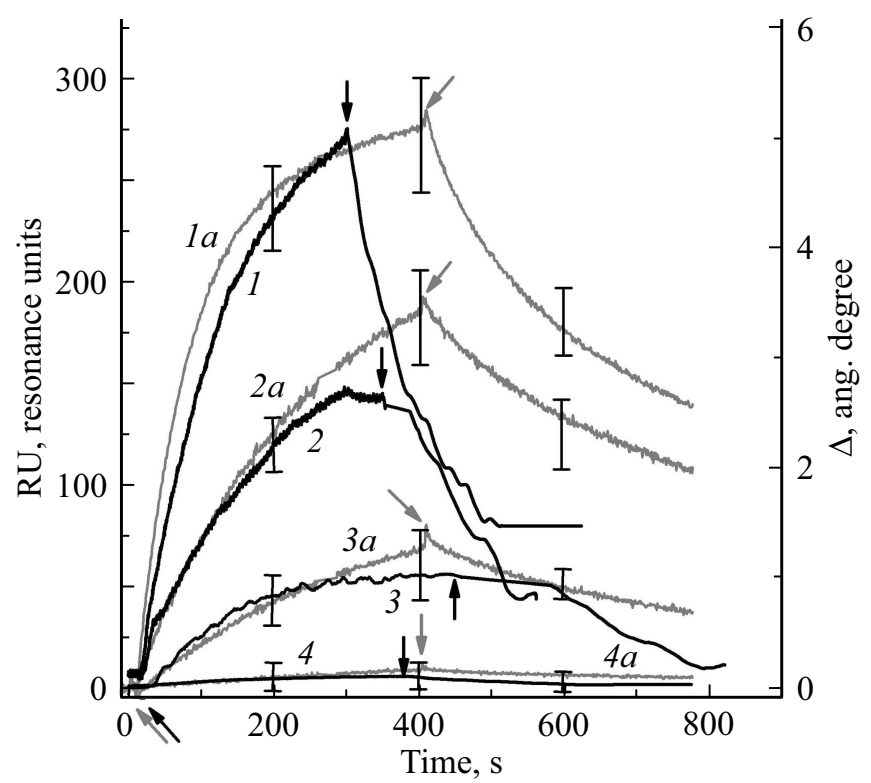

Рис. 2. Сенсограммы связывания и распада комплексов при взаимодействии антигенов раствора сыворотки крови $(1: 250)$ и высокоспецифичных моноклональных антител к опухолевой М2-ПК у пациентов с КРР и лиц группы сравнения. Стрелками показаны моменты подачи пробы и промывки ячейки. Черные кривые (1-4) - данные „Эллипс-СПЭК“, серые кривые $(1 a-4 a)$ - данные „ProteOn XPR36“. Больные с внепеченочными метастазами $(1,1 a)$, больные с метастазами в печень $(2,2 a)$, пациенты с местно-регионарным КРP $(3,3 a)$, здоровые лица $(4,4 a)$.

XPR36“ (BioRad, USA), и не уступают по чувствительности и точности зарубежным аналогам при существенно более низкой стоимости аппаратуры.

Диагностическая чувствительность детектирования для определения отличия больных КРР от здоровых лиц составила $\sim 98 \%$, что согласуется с результатами исследования [17] и превышает чувствительность методов колоноскопии, исследования кала на скрытую кровь, определения раково-эмбрионального антигена, фекальной М2-ПК $[18,19]$.

В случае, когда пороговое значение уровня сывороточной опухолевой М2-ПК составляло $11.6 \pm 4.5 \mathrm{RU}$, не было пропущено ни одного случая пациентов с КРР и можно было бы избежать $43.4 \%$ проведенных эпизодов колоноскопии. Диагностическая чувствительность для всех случаев КРР возрастала при снижении пороговых значений уровней сывороточной М2-ПК.

Данные работ, посвященных исследованию методом КР образцов сыворотки крови, показали, что основные изменения пиков, позволяющие отличать пациентов, страдающих онкологией, от здоровых лиц, происходят в диапазоне $500-1800 \mathrm{~cm}^{-1}$ [20]. В нашей работе площади пиков в диапазоне $1005-1520 \mathrm{~cm}^{-1}$ спектров КР также оказались достоверно ниже у больных КРР по сравнению со здоровыми лицами $(p<0.0001-0.05)$ (рис. 3), коррелируя со стадией процесса $(r=-0.68, p<0.001)$ и наличием метастазов $(r=-0.57, p<0.003)$. При этом соотношение интенсивностей пиков $1157 / 1520 \mathrm{~cm}^{-1}$ было наибольшим в группе сравнения с последующим достоверным снижением от группы с местно-регионарными метастазами к группе с метастазами в печень $(p<0.05-0.01)$. У пациентов с внепеченочными метастазами интенсивность всех пиков спектра оказалась минимальной (кривая 4, рис. 3 ).

Наиболее выраженное дифференцирование пациентов с различной локализацией метастазов выявлено по интенсивности пиков при 1005, 1157, $1520 \mathrm{~cm}^{-1}$ (таблица). В настоящее время в литературе нет единого мнения о принадлежности этих сигналов каким-либо конкретным химическим группам. Большинство исследователей склоняется к тому, что основной вклад в интенсивность этих пиков вносит $\beta$-каротин [20,21], вместе с этим 


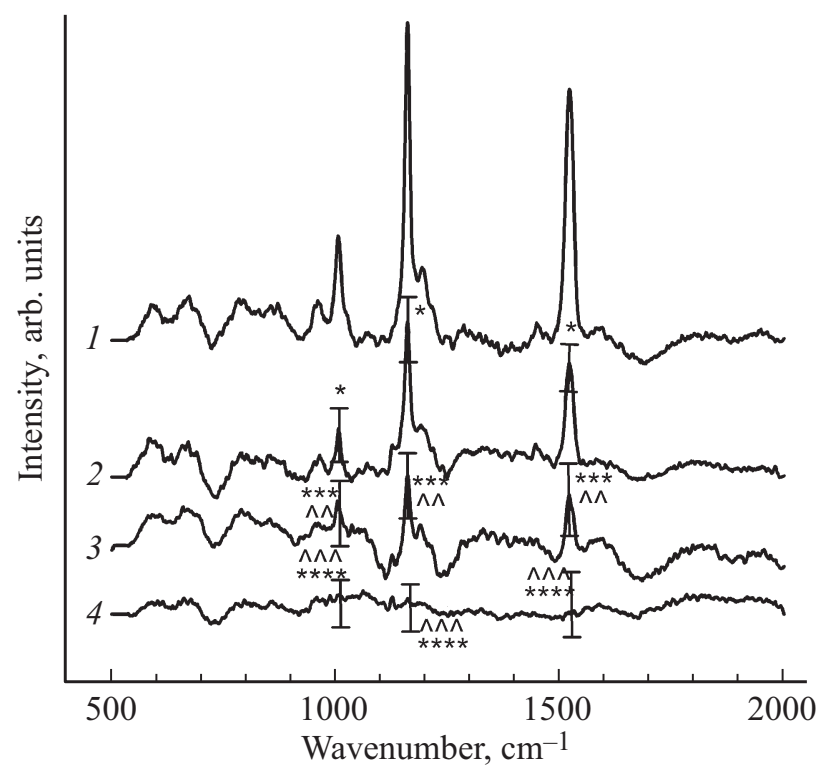

Рис. 3. Спектры комбинационного рассеяния света образцов жидкой сыворотки крови больных КРР и лиц группы сравнения, 1 - здоровые лица, 2 - пациенты с местнорегионарным КРР, 3 - больные с метастазами в печень, 4 - больные с внепеченочными метастазами. Звездочками и галочками показана статистическая значимость (таблица).

ряд авторов ассоциируют данные полосы поглощения с сигналами от фенилаланина, D-маннозы, $\alpha$-спирали и коллагена [22,23].

Различная интенсивность пиков спектров комбинационного рассеяния света в определенной степени отражает существующие различия в интегральных метаболомических профилях сывороток крови пациентов с КРР [24]. Метаболомический профиль изменяется в зависимости от локализации опухоли, и ответ хозяина на опухоль может меняться при развитии метастазирования [25]. Метастатическая болезнь биологически отлична от рака, не выходящего за пределы ткани происхождения. Многие другие агрессивные опухоли провоцируют более (или менее) бурный ответ хозяина [26], который может также отличаться из-за локального воздействия опухоли. Например, опухоль может оказывать множество паракринных эффектов на микроокружение, кроме того, метаболические или воспалительные ответы окружающих нормальных тканей могут отличаться в случаях локализации метастазов в кишечнике, печени и других органах [27]. Подобные сдвиги, вероятно, сказываются на выраженности и соотношении пиков КР спектров, демонстрирующих интегральный метаболический профиль [28], что позволяет различить пациентов с КРР с различной локализацией метастазов.

Выявленные изменения интенсивностей пиков в диапазоне 1005-1520 $\mathrm{cm}^{-1}$, обнаруженные в сыворотке крови пациентов с КРР, указывают на определенные биохимические изменения как количества, так и структуры соединений в образцах сыворотки крови при раке кишечника. Аномальный метаболизм, связанный с прогрессированием рака, возможно, вызвал эти изменения, что согласуется с результатами биохимического анализа при исследовании сыворотки крови больных с КРР [29].

Следует заметить, что прекращение выработки $\beta$ каротина в процессе развития опухоли при КРР, приводящее к уменьшению интенсивности пиков при 1005 , $1157,1520 \mathrm{~cm}^{-1}$, имеет глубинные причины, механизм его до конца не выяснен, более того, данное явление наблюдается при иных формах рака. Тем не менее, в ряде работ исследованы особенности раман-спектров у пациентов с другими онкологическими патологиями, причем выявлены отличия в иных областях спектра, чем в настоящем исследовании. Так, у пациентов с раком носоглотки [22] и раком желудка [30] выявлено снижение интенсивности пика при $632 \mathrm{~cm}^{-1}$, который ассоциируют с уменьшением относительного содержания аминокислоты тирозин (C-S-связи тирозина). Повышенный сигнал при $725 \mathrm{~cm}^{-1}$, отражающий повышенное количество оснований нуклеиновых кислот по сравнению со всеми остальными раман-активными составляющими плазмы, выявлен у больных с раком носоглотки [22], желудка [30] и шейки матки [31]. Известно, что по сравнению со здоровыми субъектами концентрация циркулирующей ДНК плазмы значительно повышена при многих солидных злокачественных новообразованиях, таких как рак носоглотки, пищевода, молочной железы, легких, печени и предстательной железы [32], что отражается на повышенном уровне сигналов при $725,1350 \mathrm{~cm}^{-1}$.

\section{Заключение}

Таким образом, представленные результаты позволяют рассматривать эллипсометрию и спектроскопию комбинационного рассеяния света как чувствительные прецизионные методы, которые можно эффективно использовать в современных биомедицинских исследованиях, в частности, в вопросах иммунологии, генной инженерии, ранней диагностики заболеваний, в том числе онкологических процессов. При проведении пилотного мониторинга обнаружены достоверные различия равновесных величин уровня отклика опухолевой М2-ПК в сыворотке крови как между группами здоровых лиц и пациентов, больных КРР, превышающие двадцатикратные, так и между группами больных КРР с метастазами различных локализаций.

Исследования, связанные с изучением особенностей раман-спектров при КРР, касались либо отличий таковых от больных с полипами кишки [24], либо различий, связанных со стадией заболевания[33]. Новизна настоящей работы заключается в выявлении особенностей раман-спектров, связанных с разной локализацией метастазов, что важно для определения дальнейшей тактики терапии (резекция одиночных метастатических очагов, паллиативные операции, адъювантная химиотерапия). 
Полученные результаты перспективны для разработки скрининговых методов ранней диагностики КРР, выявления метастазов различных локализаций, в перспективе - рецидивов заболевания, контроля за качеством проводимого лечения. Другой немаловажный факт, свидетельствующий в пользу использования данных методов в скрининговых исследованиях, связан с относительно невысокой стоимостью самих приборов и простотой методик подготовки образца и измерения.

\section{Благодарности}

Авторы выражают глубокую благодарность сотрудникам ГБУЗ НСО „НООД“, оказавшим содействие и помощь при обследовании пациентов.

\section{Финансирование работы}

Работа выполнена в рамках темы „Эпидемиологический мониторинг состояния здоровья населения и изучение молекулярно-генетических и молекулярно-биологических механизмов развития распространенных терапевтических заболеваний в Сибири для совершенствования подходов к их диагностике, профилактике и лечению“ ГЗ № 0324-2018-0001, Рег. № АAАA-А17-117112850280-2.

\section{Соблюдение этических стандартов}

Все процедуры, выполненные в исследовании с участием людей, соответствуют этическим стандартам институционального и/или национального комитета по исследовательской этике и Хельсинкской декларации 1964 года и ее последующим изменениям или сопоставимым нормам этики.

Проведение исследования одобрено Комитетом биомедицинской этики Федерального государственного бюджетного научного учреждения „Научно-исследовательский институт терапии и профилактической медицины“ (29.11.2016, протокол № 123). Все пациенты подписали информированное согласие на участие в исследовании.

\section{Конфликт интересов}

Авторы заявляют, что у них нет конфликта интересов.

\section{Список литературы}

[1] Khalilzadeh B., Shadjou N., Kanberoglu G.S., Afsharan H., de la Guardia M., Charoudeh H.N., Ostadrahimi A., Rashidi M.R. // Mikrochim. Acta. 2018. V. 185. N 9. P. 434. doi 10.1007/s00604-018-2980-6

[2] Elahi N., Kamali M., Baghersad M.H. // Talanta. 2018. V. 184. P. 537. doi 10.1016/j.talanta.2018.02.088

[3] Ogawa M., Takakura H. // Anal. Sci. 2018. V. 34. N 3. P. 273. doi 10.2116/analsci.34.273

[4] Сотников Д.В., Жердев А.В., Дзантиев Б.Б. // Успехи биологической химии. 2015. Т. 55. С. 391.
[5] Su Y., Wei Y., Zhang Y., Liu C., Nie X., Zhu Z., Liu L. // Sensors. 2018. V. 18. P. 2608. doi 10.3390/s18082608

[6] Gorodkiewicz E., Sankiewicz A., Laudański P. // Centr. Eur. J. of Chem. 2014. V. 12. N 5. P. 557. doi 10.2478/s11532-014-0512-8

[7] Chiu N.F., Kuo C.T., Lin T.L., Chang C.C., Chen C.Y. // Biosens. Bioelectron. 2017. V. 94. P. 351. doi 10.1016/j.bios.2017.03.008

[8] Yang D., Singh A., Wu H., Kroe-Barrett R. // J. Vis. Exp. 2017. V. 122. P. e55659. doi $10.3791 / 55659$

[9] Федоров В.Э., Поделякин К.А. // Медицинский альманах. 2017. T. 4. B. 49. C. 145.

[10] Pathak S., Pandanaboyana S., Daniels I., Smart N., Prasad K.R. // Surg. Oncol. 2016. V. 25. N 3. P. 246. doi 10.1016/j.suronc.2016.05.021

[11] Zaccaro C., Saracino I.M., Fiorini G., Figura N., Holton J., Castelli V., Pesci V., Gatta L., Vaira D. // Intern. Emerg. Med. 2017. V. 12. N 3. P. 333. doi 10.1007/s11739-017-1610-3

[12] Jonkheijm P., Weinrich D., Schroder H., Niemeyer C.M., Waldmann H. // Angew. Chem. Int. Ed. Engl. 2008. V. 47. P. 9618. doi 10.1002/anie.200801711

[13] Рыхлицкий С.В., Кручинин В.Н., Швеи, В.А., Спесивцев Е.В., Прокопьев В.Ю. // Приборы и техника эксперимента. 2013. № 1. С. 137. doi 10.7868/S0032816212060092

[14] Спесивцев Е.В., Рыхлицкий С.В., Швеи, В.А. Эллипсометр. Патент на изобретение № 2303623, 2007.

[15] Швец, B.A., Спесивщев Е.В., Рыхлищкий С.В. // Опт. и спектр. 2004. Т. 97. № 3. С. 514.

[16] Tompkins H., Irene E.A. Handbook of ellipsometry. William Andrew Publishing, Springer, 2005. 789 p.

[17] Kikawada M., Ono A., Inami W., Kawata Y. // Anal. Chem. 2016. V. 88. N 2. P. 1407. doi 10.1021/acs.analchem.5b04060

[18] Meng W., Zhu H.H., Xu Z.F., Cai S.R., Dong Q., Pan Q.R., Zheng S., Zhang S.Z. // World J. Gastrointest. Oncol. 2012. V. 4. N 6. P. 145. doi 10.4251/wjgo.v4.i6.145

[19] Abdullah M., Rani A.A., Simadibrata M., Fauzi A., Syam A.F. // Acta Med. Indones. 2012. V. 44. N 2. P. 94.

[20] Sahu A., Sawant S., Mamgainc H., Krishna C.M. // Analyst. 2013. V. 138. N 14. P. 4161. doi 10.1039/c3an00308f

[21] Medipally D.K., Maguire A., Bryant J., Armstrong J., Dunne M., Finn M., Lyng F.M., Meade A.D. // Analyst. 2017. V. 142. N 8. P. 1216. doi 10.1039/C6AN02100J

[22] Feng S., Chen R., Lin J., Pan J., Chen G., Li Y., Cheng M., Huang Z., Chen J., Zeng H. // Biosens. Bioelectron. 2010. V. 25. N 11. P. 2414. doi 10.1016/j.bios.2010.03.033

[23] Movasaghi Z., Rehman S., Rehman I.U. // Appl. Spectrosc. Rev. 2007. V. 42. N 5. P. 493. doi 10.1080/05704920701551530

[24] Feng S., Wang W., Tai I.T., Chen G., Chen R., Zeng H. // Biomedical Optics Express. 2015. V. 6. N 9. P. 3494. doi 10.1364/BOE.6.003494

[25] Zhou Q., Peng R.Q., Wu X.J., Xia Q., Hou J.H., Ding Y., Zhou Q.M., Zhang X., Pang Z.Z., Wan D.S., Zeng Y.X., Zhang X.S. // J. Transl. Med. 2010. V. 8. P. 13(1-9). doi 10.1186/1479-5876-8-13

[26] Giusca S.E., Zugun F.E., Tarcoveanu E., Carasevici E., Amalinei C., Caruntu I.D. // Rom. J. Morphol. Embryol. 2010. V. 51. N 1. P. 73.

[27] Meyerhardt J.A., Tepper J.E., Venook A. // J. Clin. Oncol. 2015. V. 33. N 16. P. 1717. doi 10.1200/JCO.2015.60.8661 
[28] Kong K., Kendall C., Stone N., Notingher I. // Advanced Drug Delivery Reviews. 2015. V. 89. P. 121. doi 10.1016/j.addr.2015.03.009

[29] Lin D., Feng S., Pan J., Chen Y., Lin J., Chen G., Xie S., Zeng H., Chen R. // Opt. Express. 2011. V. 19. N 14. P. 13565. doi 10.1364/OE.19.013565

[30] Feng S., Chen R., Lin J., Pan J., Wu Y., Li Y., Chen J., Zeng H. // Biosens. Bioelectron. 2011. V. 26. N 7. P. 3167. doi 10.1016/j.bios.2010.12.020

[31] Feng S., Lin D., Lin J., Li B., Huang Z., Chen G., Zhang W., Wang L., Pan J., Chen R., Zeng H. // Analyst (Lond.). 2013. V. 138. N 14. P. 3967. doi 10.1039/c3an36890d

[32] Banki F., Yacoub W.N., Hagen J.A., Mason R.J., Ayazi S., DeMeester S.R., Lipham J.C., Danenberg K., Danenberg P., DeMeester T.R. // J. Am. Coll. Surg. 2008. V. 207. N 1. P. 30. doi 10.1016/j.jamcollsurg.2008.01.004

[33] Li P., Chen C., Deng X., Mao H., Jin S. // J. Biomed. Opt. 2015. V. 20. P. 037004 (7). doi 10.1117/1.JBO.20.3.037004 